\title{
Tempospect Theory of Intertemporal Choice
}

\author{
Taiki Takahashi, Ruokang Han \\ Department of Behavioral Science, Center for Experimental Research in Social Sciences, \\ Hokkaido University, Hokkaido, Japan \\ Email: taikitakahashi@gmail.com
}

Received May $3^{\text {rd }}, 2012$; revised June $5^{\text {th }}, 2012$; accepted July $6^{\text {th }}, 2012$

\begin{abstract}
Anomalies in intertemporal choice (e.g. hyperbolic discounting, subadditive discounting, a sign effect, a magnitude effect, and a delay-speedup asymmetry) have been investigated in neuroeconomics and behavioral neuroeconomics. In this study we propose a "tempospect" theory of intertemporal choice which can account for these anomalies in intertemporal choice. The key features of the present theory are: 1) decision over time is made with psychological time; and 2) psychological time is determined by a change in delay until receipt (i.e., positive or negative time-interval between options); 3) psychological time is less sensitive to a decrease in delay in comparison to an increase in delay; and 4) psychological time is influenced by the sign and magnitude of the delayed outcomes. Implications of the present theory for neuroeconomics are discussed.
\end{abstract}

Keywords: Time Discounting; Psychological Time; Psychophysics; Behavioral Economics; Neuroeconomics

\section{Introduction}

People tend to devalue the subjective value of outcomes as delay until its receipt increases, which is referred to as temporal discounting (intertemporal choice). Studies in behavioral economics (Loewenstein \& Prelec, 1992; Frederick et al., 2002; Read \& Roelofsma, 2003; Scholten \& Read, 2010) and neuroeconomics (McClure et al., 2004; Kable \& Glimcher, 2007; Takahashi, 2009) demonstrated several anomalies in intertemporal choice (temporal discounting). Neuropsychopharmacological studies indicate that these anomalies are related to addiction (Bickel \& Marsch, 2001). Since its introduction by Samuelson (1937), an exponential discounting model for intertemporal choice has dominated in economic theory. In exponential discounting, people are assumed to be time-consistent, because time-discount rate is constant over time. However, later empirical evidence suggests that several anomalies exist in human and animal intertemporal choice behavior (Thaler, 1981; Loewenstein \& Prelec, 1992; Frederick et al., 2002). The important anomalies are 1) hyperbolic discounting; 2) subadditive discounting; 3) a sign effect; 4) a magnitude effect; and 5) a delay-speedup asymmetry (see Loewenstein \& Prelec, 1992; Frederick et al., 2002; Scholten \& Read, 2010, for a review). We briefly explain these anomalies below.

\section{Hyperbolic Discounting}

Time preferences between two delayed outcomes often switch when both delays are increased by a given constant time-interval. For instance, a person might prefer one apple today to two apples tomorrow, but at the same time, prefer two apples in 101 days to one apple in 100 day. This behavioral tendency gives rise to time-inconsistent behavior (Strotz, 1956). In other words, people tend to make patient plans in the distant future, but make impulsive (impatient) actions in the near future. It is to be noted that in exponential discounting, this type of time-inconsistency does not exist because the time-discount rate is constant over time. Recently, McClure et al. (2004) and Kable and Glimcher (2007) examined the neural correlates of hyperbolic discounting.

\section{Subadditive Discounting}

Similar to human probability judgment, temporal discounting is subadditive. Consider someone judging the present value of an outcome to be received in one month. He or she can separately discount for each of the four weeks in the month, or discount once for the unbroken one month. Subadditive discounting (Read, 2001) means that the total discounting is greater when the month is divided into weeks. This anomaly is a sharp evidence against Samuelson's discounted utility theory (Scholten \& Read, 2010).

\section{Sign Effect}

Empirical studies reported that loss is less steeply time-discounted than gain (Thaler, 1981). In Thaler (1981)'s study, time-discount rates for gains were three to ten times greater than those for losses. A more recent study also found the sign effect in temporal discounting (Estle et al., 2006).

\section{Magnitude Effect}

Behavioral economic studies revealed that larger gains are less steeply time-discounted than smaller ones (Thaler, 1981; Estle et al., 2006). Similar to the sign effect in intertemporal choice, this anomaly challenges the assumptions in Samuelson's discounted utility model (Loewenstein \& Prelec, 1992; Frederick et al., 2002).

\section{Delay-Speedup Asymmetry}

Loewenstein (1988) demonstrated that time-discount rates can be dramatically affected by whether the change in delivery 
time of an outcome is framed as an acceleration ("speedup") or a "delay" from a certain temporal reference point. For instance, people who didn't expect to receive a product for another year would pay an average of $\$ 54$ to receive it immediately, but those who thought they would receive it immediately demanded an average of $\$ 126$ to delay its receipt by a year. In other words, time-discount rate is larger for "delay" than for "speedup" of receiving the delayed reward. This anomaly also contradicts with the assumptions in discounted utility theory (Loewenstein \& Prelec, 1992; Frederick et al., 2002).

Taken together, there has been accumulating evidence against the standard exponentially discounted utility model. To date, however, no theory is capable of accounting for these important anomalies, although some behavioral economists proposed descriptive models of intertemporal choice (Frederick et al., 2010; Scholten \& Read, 2010). In this study, we propose a model which can explain all of the anomalies in intertemporal choice by incorporating nonlinear and delay-speedup asymmetrical subjective time in decision over time.

\section{Tempospect Theory for Intertemporal Decision-Making}

\section{Temporal Cognition in Intertemporal Choice}

In order to account for the anomalies in intertemporal choice, various theoretical models have been proposed (Loewenstein \& Prelec, 1992; Takahashi, 2005; Takahashi, 2006; Takahashi, 2009; Scholten \& Read, 2006; Scholten \& Read, 2010). Generally speaking, Loewenstein and Prelec's theory is a value-based account of the anomalies in intertemporal choice, in contrast, Scholten and Read's group and Takahashi's theories are timebased accounts. In Takahashi's theory, both hyperbolic (Takahashi, 2005) and subadditive discounting is due to nonlinearity in psychological perception (weighting) of delay or time-interval between options. Specifically, time-interval in the distant future is perceived as a shorter psychological time in comparison to that in the near future, which can explain why people are patient regarding the distant future but impatient regarding the near future (hyperbolic discounting). Zauberman et al. (2009) experimentally confirmed the predictions from Takahashi's nonlinear time-perception theory of hyperbolic and subadditive discounting. However, neither Loewenstein-Prelec theory, Scholten-Read theory, nor Takahashi's theory totally explains all the anomalies mentioned earlier. In order to explain all the anomalies mentioned, the present study generalizes the timebased accounts proposed by Scholten-Read and Takahashi. In generalizing the time-based accounts, we specify the functional forms of psychological time and temporal discounting, in order to future parametric applications in behavioral economics and neuroeconomics, although the main conclusions of the present study do not depend on the precise functional forms.

\section{Time Discounting with Psychological Time}

Takahashi (2005) proposed that subjects utilize the following psychological time in their intertemporal choice:

$$
\tau(D)=\alpha \ln (1+\beta D) .
$$

where $\tau(D)$ is subjective time (or time-weighting) of delay $D$ when the delayed outcome is obtained. If we further assume that subject discount the delayed outcome $x$ exponentially with the psychological time, we obtain

$$
V(x, D)=V(x, 0) \exp (-k \tau(D)) .
$$

where $V(x, D)$ is the time-discounted value of the delayed outcome $x$ obtained at delay $D$. Equation (2) is hyperbolic in physical delay $D$ (Takahashi, 2005). It is to be noted that Equation (2) is equivalent to Loewenstein and Prelec's generalized hyperbola (Loewenstein \& Prelec, 1992) and the "q-exponential" time-discount model developed in econophysics (Cajueiro, 2006; Takahashi et al., 2007; Takahashi, 2007; Takahashi et al., 2008; Destefano \& Martinez, 2011). Takahashi (2005) claimed that Equation (2) can account for decreasing impatience (hyperbolic discounting) over physical time, although the discount rate with respect to psychological time $(k>0)$ is independent of psychological time, and Zauberman et al. (2009) confirmed this claim in their behavioral economic experiment.

\section{Tempospect Theory of Intertemporal Choice}

Scholten and Read (2010) and Takahashi (2006) stated that intertemporal choice occurs with respect to time-intervals rather than delay (time-point) per se at which the delayed outcome is obtained. Therefore, it is natural to generalize Equation (1) to the following equations:

$$
\begin{gathered}
\Delta \tau(\Delta D)=\alpha_{d} \ln \left(1+\beta_{d} \Delta D\right) \quad(\text { for } \Delta D>0) . \\
\Delta \tau(\Delta D)=\alpha_{s} \ln \left(1+\beta_{s}[-\Delta D]\right) \quad(\text { for } \Delta D<0) .
\end{gathered}
$$

where $\Delta \tau(\Delta D)$ is subjective time-interval (what we call "tempospect") between options which are obtained at the physical time-interval of $\Delta D(\Delta D>0$ and $\Delta D<0$ correspond to "delay" and "speedup" of the delayed option, respectively). Here we make a natural assumption, as is the case with the gain-loss asymmetrical value function in Kahneman-Tversky's prospect thery (Kahneman \& Tversky, 1979; Tversky \& Kahneman, 1992) in which an increase in outcomes less dramatically impacts subjective valuation than a decrease in outcomes (referred to as "loss aversion"), that $\Delta \tau$ is larger for "delay" (an increase in delay until receipt, which may be perceived as "loss") than "speedup" (a decrease in delay until receipt, which may be perceived as "gain"), when the outcome is positive (gain). Oppositely, $\Delta \tau$ may be smaller for "dalay" than for "speedup" when the outcome is negative (loss), because an increase in delay in waiting for loss may be perceived as gain. Under these assumptions, the time-interval discount function may be:

$$
\begin{gathered}
V(x, t+\Delta D)=V(x, t) \exp (-k \Delta \tau(\Delta D)) \quad(\text { for } \Delta D>0) . \\
V(x, t+\Delta D)=V(x, t) \exp (k \Delta \tau(\Delta D)) \quad(\text { for } \Delta D<0) .
\end{gathered}
$$

where $V(x, t+\Delta D)$ is the subjective value of the delayed outcome obtained at delay $t+\Delta D$. As can be seen from Equation (5), when $\Delta \tau$ is large, the subjective value of the outcome is small. Considering the assumption that $\Delta \tau$ may be larger for "delay" than "speedup" in waiting for delayed gain, we can suppose that subjective value of the delayed reward changes more dramatically when the option is delayed than sped up. Concerning the characteristics of the outcome $x$, when $x$ is negative (loss), $\Delta D$ may have smaller impact on decision over time, than when $x$ is positive (gain), because subjective valuation of the outcome, rather than $\Delta D$, may have stronger impact when $x$ is negative, due to loss aversion in the value function in prospect theory (Kahneman \& Tversky, 1979; Tversky \& Kahneman, 1992). This can explain the sign effect in intertemporal 
choice. Also, when the outcome is large, $\Delta D$ may have smaller impact on decision over time, again the subjective value of the outcome, rather than $\Delta D$, may have stronger impact. This may account for the magnitude effect in intertemporal choice. Taken together, our present "tempospect" theory can account for the important anomalies in intertemporal choice.

\section{Implications for Behavioral Economics and Neuroeconomics}

Since Loewenstein and Prelec (1992)'s proposal, studies in intertemporal choice have mainly focused on the value-based account of the anomalies in intertemporal choice. Subsequent studies examined the roles of the shape of value functions which can explain the anomalies in intertemporal choice (AlNowaihi \& Dhami, 2006; Al-Nowaihi \& Dhami, 2009). Our present study emphasizes, in line with perspectives proposed by Scholten and Read's group (Scholten \& Read, 2006, 2010), the roles of psychological time regarding time-intervals between options. By estimating the functional forms of the psychological time for time-intervals, we will be able to establish more precise functional forms of temporal discounting. Also, investigations into neural processing underlying psychological time in decision over time may help establish more effective medical treatments for addiction and other impulsive and problematic behaviors observed in psychiatric illnesses (Takahashi, 2009, for a review).

\section{REFERENCES}

Al-Nowaihi, A., \& Dhami, S. (2006). A note on the Loewenstein-Prelec theory of intertemporal choice. Mathematical Social Sciences, 52, 99-108. doi:10.1016/j.mathsocsci.2006.04.001

Al-Nowaihi, A., \& Dhami, S. (2009). A value function that explains the magnitude and sign effects. Economics Letters, 105, 224-229. doi:10.1016/j.econlet.2009.08.004

Bickel, W. K., \& Marsch, L. A. (2001). Toward a behavioral economic understanding of drug dependence: Delay discounting processes. Addiction, 96, 73-86. doi:10.1046/j.1360-0443.2001.961736.x

Cajueiro, D. (2006). A note on the relevance of the q-exponential function in the context of intertemporal choices. Physica A, 364, 385-388. doi:10.1016/j.physa.2005.08.056

Destefano, N., \& Martinez, A. (2011). The additive property of the inconsistency degree in intertemporal decision making through the generalization of psychophysical laws. Physica A, 390, 1763-1772. doi:10.1016/j.physa.2011.01.016

Estle, S. J., Green, L., Myerson, J., \& Holt, D. (2006). Differential effects of amount on temporal and probability discounting of gains and losses. Memory and Cognition, 34, 914-928. doi:10.3758/BF03193437

Frederick, S., Loewenstein, G., \& O’Donoghue, T. (2002). Time discounting and time preference: A critical review. Journal of Economic Literature, 40, 351-401. doi:10.1257/002205102320161311
Kable, J., \& Glimcher, P. (2007). The neural correlates of subjective value during intertemporal choice. Nature Neuroscience, 10, 16251633. doi: $10.1038 / \mathrm{nn} 2007$

Kahneman, D., \& Tversky, A. (1979). Prospect theory-Analysis of decision under risk. Econometrica, 47, 263-291. doi: $10.2307 / 1914185$

Loewenstein, G. (1988). Frames of mind in intertemporal choice. Management Science, 34, 200-214. doi:10.1287/mnsc.34.2.200

Loewenstein, G., \& Prelec, D. (1992). Anomalies in intertemporal choice: Evidence and Interpretation. Quarterly Journal of Economics, 107, 573-597. doi:10.2307/2118482

McClure, S., Laibson, D., Loewenstein, G., \& Cohen, J. (2004). Separate neural systems value immediate and delayed monetary rewards. Science, 306, 503-507. doi:10.1126/science.1100907

Read, D. (2001). Is time-discounting hyperbolic or subadditive? Journal of Risk Uncertainty, 23, 5-32. doi:10.1023/A:1011198414683

Read, D., \& Roelofsma, P. (2003). Subadditive versus hyperbolic discounting: A comparison of choice and matching. Organizational Behavior and Human Decision Processes, 91, 140-153. doi:10.1016/S0749-5978(03)00060-8

Samuelson, P. (1937). A note on measurement of utility. Review of Economic Studie, 4, 155-161. doi:10.2307/2967612

Scholten, M., \& Read, D. (2010). The psychology of intertemporal tradeoffs. Psychological Review, 117, 925-944. doi: $10.1037 / \mathrm{a} 0019619$

Scholten, M., \& Read, D. (2006). Discounting by intervals: A generalized model of intertemporal choice. Management Science, 52, 1424-1436. doi: $10.1287 / \mathrm{mnsc} .1060 .0534$

Strotz, R. H. (1955). Myopia and inconsistency in dynamic utility maximization. Review of Economic Studies, 23, 165-180. doi: $10.2307 / 2295722$

Takahashi, T. (2005). Loss of self-control in intertemporal choice may be attributable to logarithmic time-perception. Medical Hypotheses, 65, 691-693. doi:10.1016/j.mehy.2005.04.040

Takahashi, T. (2006). Time-estimation error following Weber-Fechner law may explain subadditive time-discounting. Medical Hypotheses, 67, 1372-1374. doi:10.1016/j.mehy.2006.05.056

Thaler, R. H. (1981). Some empirical evidence on dynamic inconsistency. Economics Letters, 8, 201-207. doi:10.1016/0165-1765(81)90067-7

Takahashi, T. (2007). A comparison of intertemporal choices for oneself versus someone else based on Tsallis' statistics. Physica A, 385, 637-644. doi:10.1016/j.physa.2007.07.020

Takahashi, T., Oono, H., \& Radford, M. (2007). Empirical estimation of consistency parameter in intertemporal choice based on Tsallis' statistics. Physica A, 381, 338-342. doi:10.1016/j.physa.2007.03.038

Takahashi, T. (2009). Theoretical frameworks for neuroeconomics of intertemporal choice. Journal of Neuroscience, Psychology, and Economics, 2, 75-90. doi:10.1037/a0015463

Tversky, A., \& Kahneman, D. (1992). Advances in prospect theorycumulative representation of uncertainty. Journal of Risk and Uncertainty, 5, 297-323. doi:10.1007/BF00122574

Zauberman, G., Kim, B., Malkoc, S., \& Bettman, J. (2009). Discounting time and time discounting: Subjective time perception and intertemporal preferences. Journal of Marketing Research, 46, 543556. doi:10.1509/jmkr.46.4.543 